Planetary Systems in the Universe - Observation, Formation and Evolution

Proceedings IAU Symposium No. 202, (c)2004 IAU

Alan Penny, Pawel Artymowicz, Anne-Marie Lagrange, 8 Sara Russell, eds.

\title{
The Drake equation may need new factors based on peculiarities of planets of Sun-like stars
}

\author{
L. V. Ksanfomality \\ Space Research Institute, Moscow, Russia
}

\begin{abstract}
.
$N=R_{s} f_{p} n_{e} f_{l} f_{i} f_{c} L$. This well known Drake equation denoting the number $\mathrm{N}$ of civilizations in the Galaxy, includes a factor $f_{p}$ that is the fraction of stars that have planetary systems, and another factor ne that is the mean number of planets suitable for life. The latter is usually understood as the number of planets within the habitable zone. When the Solar system remained a unique object of its kind the sense of $f_{p}$ and $n_{e}$ seemed to be clear. There is now a limited statistic in hand about the features of other planetary systems, permitting a rough estimation of $f_{p}$ and $n_{e}$ (based on the list of 32 star companions, compiled by G. Marcy, 14.02.2000).
\end{abstract}

\section{Introduction}

The development of the concept that the Solar system is a representative planetary system in the Galaxy is a consequence of the fact that, before the 10th decade of the twentieth century, the Solar system was the only known object of its kind. As a result, studies of the known planets produced a certain stereotype in theoretical investigations. This is why the discovery of extrasolar planetary systems changes our basic notions of the criteria and physics of normal planets. About 20 companions have been found amid 500 main sequence Sun-like stars that have a mass more or less close to that of Jupiter. One may assume that $f_{p s}=0.04-0.05$ for solar type stars. Does this mean that the $f_{p}$ and $n_{e}$ factors are already known?

The $f_{p}$ and $n_{e}$ factors were cumulative characteristics permitting Drakes equation to be used without knowing anything about the exoplanets physics. What is known now permits a stricter approach, that splits $f_{p}$ and $n_{e}$ into minor sub-factors (and raises new problems). For example, should brown dwarfs orbiting a star be taken as planets, and do they make up a part of the $f_{p}$ factor? The goal of this paper is to consider the impact of characteristics of extrasolar planets on the components of the Drake equation and to obtain an estimate, where possible.

There is a striking difference in the structure of the Solar system and that of planetary systems of other stars that have been discovered to date (Mayor et al., 1998). The now known distribution of extrasolar planets by semimajor axis and masses show that the Solar system is scarcely a representative planetary system in the Galaxy for estimation of the $f_{p}$ and $n_{e}$ factors.

The very existence of the hot jupiter type exoplanets may be considered in the light of new theoretical works that meet significant problems producing 


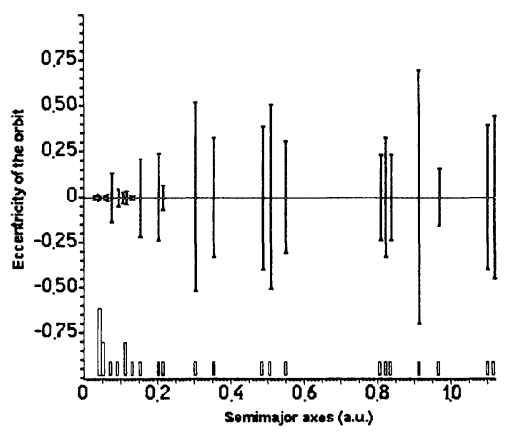

Figure 1. Eccentricities of the considered exoplanetary orbits. The position of the line 0 above the horizontal axis is the normalized major semiaxis of each orbit. The bar size indicates the limits of the planets wandering in the system due to the orbital eccentriicty.

Jupiter in its real orbit. However the growth of Jupiter was an essential factor in the Solar systems history. Two major waves of impacts, 4.5 and 3.8 billion years ago, played an important role. Large impact events are documented in planets surfaces from accretion processes. Eventually, a steady (low entropy) state was reached in the Solar system. Jupiter and other giant planets having stable and almost circular orbits protected the inner planets against heavy bombardment, diminishing its rate by many orders of magnitude. (There are even remakes of the anthropic principal, stating that the onset of terrestrial life is obliged to Jupiter).

As for the exoplanets, almost all of those orbiting beyond $0.2 \mathrm{AU}$, have an eccentricity of orbit greater than 0.2 , typically $0.3-0.5$ (Fig.1). Together with their possible migration inwards in the system, this may be a special problem for inner planets existence and for the $f_{p}$ factor value.

The recent observations of very young stars demonstrates that the giant planets contain no more than a few per cent of the hydrogen that should have been contained in an early protoplanetary disk (Zukerman et al., 1995). It is likely that the star loses the gas during the unstable first few million years of their life. But it takes about a hundred million years for Jupiter to grow to its 318 Earth masses, a hundred million years when there is already no hydrogen for it, which inhibits giant planet formation. A possible conclusion could be that the special unusual conditions necessary to form Jupiter and Saturn occurred once, leading to the existent Solar systems structure, and that planetary systems rarely include distant gas giant planets similar to those in the Solar system (Wetherill, 1995).

Together with this conclusion a giant planet may assemble wholesale, without a long process of a core formation, when a dense clump of gas within the protoplanetary disk suddenly collapsed (Boss, 1997; Rasio and Ford, 1996). A possible instability in the protoplanetary disk could lead to the formation of giant gaseous protoplanets within orbits of hypothetical terrestrial planets, leaving them unprotected. 


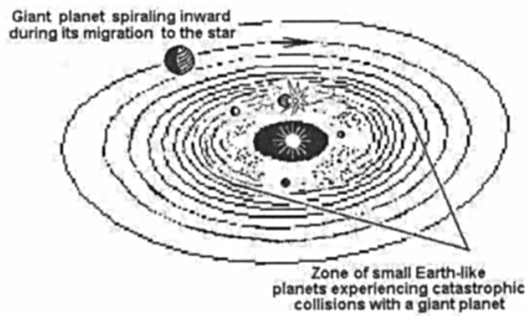

Figure 2. A collision of an migrating giant planet having a high orbital eccentricity, with a small Earth-type planet, wiping the small planet out, is just a question of time.

Taking into account all these mechanisms one should divide $f_{p}$ into few different factors, for systems of inner planets protected by giant planets $f_{p p}$, or unprotected $\left(1-f_{p p}\right)$, against heavy meteoritic bombardment. The Solar system and may be two companions from the list of 30 other known stars with planets (47UMa and $\iota$ Hor), are protected systems, meaning that $f_{p p}$ is less than 0.1 , assuming that terrestrial planets do exist in the systems. The validity of this last hypothesis for the moment would be most important but has no proof (except for the Solar system), and the coefficient $f_{p t}$ for presence of terrestrial planets remains unknown.

The Solar system is in a remarkably low entropy state. High entropy planetary systems may be the norm (Marcy et al., 1999). A low entropy system may be indicated by a factor $f_{p e}$. Besides the Solar system they are the hot jupiters systems. So the ftor $f_{p e}$ could be about 0.3 .

All planet-bearing stars systematically have high metallicity, with a criteria $[\mathrm{Fe} / \mathrm{H}]=0.1-0.2$ (Gonzales, 1999). This means that all they are rich in heavy elements compared to stars in the solar neighborhood, as is the Sun. One may conclude that the two previous generations of stars could not possess planets (Ksanfomality, 2000). This also means that planet formation depends on the chemical composition of the protoplanetary disk that additionally affects $f_{p}$. A rough estimation of $f_{p m}$ (for the metallicity) may be 0.04-0.05 for stars of spectral class G, same as given in the beginning of the paper. (Late spectral classes stars are of no interest for the Drake equation factors, see Ksanfomality, 1998).

About a half of the discovered exoplanets are of "hot jupiter" type. They are giants of a few jovian masses, placed in a very low orbit and with an orbital period 3-14 days (Marcy et al., 1998). Their equilibrium temperature exceeds $1000 \mathrm{~K}$. This is crucial in diminishing another of Drakes factors, $n_{e}$, as this kind of planet is not suitable for life. The systems with "hot jupiters" make up approximately $1 / 2$ of all known objects. The factor $f_{p d}$ denoting a system with normal distant planets is about 0.5 .

There could be many tens of protojupiters subject to migration during a planetary system formation. The migration models rely on an interaction between the protoplanetary disk and the giant planet, and on a possible interaction of two giant planets during a close encounter, forcing the planet to spiral inward. 
If the migrating giant planet has a high orbital eccentricity (Fig.1), a collision, wiping the small planet out, is just a question of time. In its migration a protoplanet or a giant planet scans space and destroys small Earh-like planets. The giant planet migrating inwards gradually empties the inner part of the planetary system (Fig.2) in the same way as Neptunes satellite Triton once cleaned the vicinity of Neptune. The absence of migration may be introduced by a special factor $f_{p x}$ that is highly important, but remains unknown. The $f_{p}$ factor depends crucially on migration phenomena and high orbital eccentricities in a planetary system. Of the 12 distant planets under consideration only 3 have the low orbital eccentricities. It is unknown how many of them are subject to migration. Anyway, the low orbital eccentricity $f_{p e}$ factor is less than 0.25 .

\section{Conclusions}

Very rough estimates have been made for different components making up the $f_{p}$ and $n_{s}$ factors of the Drake equation. The results are the following.

The protection of inner planets against meteoritic bombardment $f_{p p}$ is $<0.1$.

The factor $f_{p d}$ denoting systems with normal distant planets is 0.5 or less.

The high metallicity star factor $f_{p m}$ may be about 0.05 for G spectral class stars.

The low orbital eccentricity $f_{p e}$ factor is less than 0.25 .

The factor $f_{p e}$, that represents low entropy planetary systems may be about 0.3 . The coefficient describing presence of terrestrial planets $f_{p t}$ remains unknown.

The migration absence factor $f_{p x}$ remains unknown.

Optimistically assuming that two last factors are about $0.5\left(f_{p t}=f_{p x}=\right.$ 0.5 ), and remembering that Sun-like stars make up about $6 \%$ of all stars of the main spectral sequence, one obtains the product of all the factors $f_{p}=2.8$ ?10-6. This means that amid the $10^{11}$ stars of the Galaxy there may be only about 30,000 stars with planetary systems that meet our notion of the physics of terrestrial planets. It may turn out that the familiar planets in our Solar system are the strangers of the Galaxy.

\section{References}

Boss, A. 1997, Science, 276, 1836

Gonzales, G. 1999, MNRAS, 308, 447

Ksanfomality, L. 2000, Solar System Research, 34, 495

Ksanfomality, L. 1998, Planetary Systems: the Long View Eds Celnikier L.M. and Tran Thanh Van J., France. Frontieres, 481

Mayor, M., Queloz D., Udry S. \& Halbwachs J.-L. 1998, Planetary Systems: the Long View, Eds Celnikier, L.M. and Tran Thanh Van, J., France. Frontieres, 339

Marcy, G.W., Butler, P., Vogt, S.S., \& Apps, K. 1998, PASP110, 1389

Marcy G.W., Butler P., Vogt S., et al. 1999, Bioastronomy 99 Conference (Hawaii, 2-6 August 1999), Abstracts, 95

Rasio F.A., Ford E.B. 1996, Science, 274, 954

Wetherill, G.W. 1995, Nature, 373, 470

Wetherill, G.W. 1996, Science, 274, 954

Zuckerman, B., Foveille, T., \& Kastner J.H. 1995, Nature, 373, 494 